\title{
An Examination of Strength and Concentric Work Ratios During Variable Range of Motion Training
}

\author{
au1 Ross A. Clark, ${ }^{1,2}$ Adam L. Bryant, ${ }^{2}$ and Brendan Humphries ${ }^{1}$ \\ ${ }^{1}$ School of Health and Human Performance, Faculty of Arts, Health and Science, Central Queensland University, \\ North Rockhampton, Queensland; ${ }^{2}$ Centre for Health, Exercise and Sports Medicine, School of Physiotherapy, \\ Faculty of Medicine, Dentistry and Health Sciences, The University of Melbourne, Melbourne, Victoria, Australia
}

\begin{abstract}
Clark, RA, Bryant, AL, and Humphries, B. An examination of strength and concentric work ratios during variable range of motion training. J Strength Cond Res 22: xxx-xxx, 2008-Variable range of motion $(\mathrm{ROM})$ training consists of partial $\mathrm{ROM}$ resistance training with the countermovement being performed at a different phase of the movement for each set. In this study, we assessed the effect of this method of training on peak force, load lifted, and concentric work performed. Six male subjects with resistance training backgrounds (age $20.2 \pm 1.3$ years, height $179.4 \pm 4.6$ $\mathrm{cm}$, weight $89.6 \pm 9.9 \mathrm{~kg}$, 6-repetition maximum [6RM] bench press $92.5 \pm 14.3 \mathrm{~kg}$ ) participated in this study. Testing consisted of $6 \mathrm{RM}$ bench press strength tests during full (FULL), three quarter $(3 / 4)$, one half $(1 / 2)$, and one quarter ( $1 / 4)$ ROM from full elbow extension bench press performed on a Smith machine. The 6RM load, peak force (PF), and concentric work (W) performed during each ROM was examined using a one-way analysis of variance performed at an $\alpha$ level of $p<0.05$. The 6RM load increased significantly as the ROM was decreased for all tests (FULL $=92.5$ $\pm 14.3 \mathrm{~kg}, 3 / 4=102.1 \pm 14.3 \mathrm{~kg}, 1 / 2=123.3 \pm 23.6 \mathrm{~kg}, 1 / 4=$ $160.9 \pm 26.2 \mathrm{~kg})$. PF during each test was significantly higher during the $1 / 4(1924.8 \pm 557.9 \mathrm{~N})$ and $1 / 2(1859.4 \pm 317.1 \mathrm{~N})$ ROM from full elbow extension bench press when compared with the $3 / 4(1242.2 \pm 254.6 \mathrm{~N})$ and FULL $(1200.5 \pm 252.5 \mathrm{~N})$ ROM exercise. Although higher force levels were evident, the restriction in barbell displacement resulted in a subsequent reduction in $\mathrm{W}$ as the lifting ROM was reduced. These results suggest that variable ROM resistance training results in increased force production as the ROM diminishes.
\end{abstract}

KEY WoRDS deceleration phase, partial range of motion, strength transfer

Address correspondence to: Dr. Ross A. Clark, raclark@unimelb.edu.au. $22(5) / 1-4$

Journal of Strength and Conditioning Research

(c) 2008 National Strength and Conditioning Association

\section{INTRODUCTION}

A resistance training program utilizing the full range of motion (ROM) may not be optimal for enhancing muscle force levels. In this respect, previous studies have shown that full ROM exercises consist of a large deceleration phase $(2,5,9)$, resulting in a substantial proportion of the movement being performed at force levels far below maximal. What makes this submaximal performance during the exercise so detrimental from an athlete's point of view is that it occurs toward the terminal range of the movement (ROM), which is often the critical phase for athletic performance.

In addition, athletes are often required to perform countermovements at various joint angles during sport (1). For this reason, high-level sprinters are instructed to perform half (1/2) ROM squats to improve acceleration and one quarter $(1 / 4)$ ROM squats to enhance maximal speed (10). Therefore, an athlete participating in a sport that requires optimal countermovement performance at various phases of the ROM may benefit from a resistance training program that replicates these movements.

Performing variable ROM (VROM) training, which consists of the countermovement performed during each set taking place at a different joint angle, may further increase the sports specificity of resistance training. For example, if the athlete was to perform four sets of bench presses, the countermovement for each individual set may be performed at full, three quarter $(3 / 4), 1 / 2$, and $1 / 4$ ROM of the exercise (see Figure 1). In this case, the full ROM set would consist of the barbell being lowered to the chest before being lifted to full elbow extension. In contrast, for the $3 / 4 \mathrm{ROM}$ set, the barbell would only be lowered three quarters of the distance from full elbow extension to the chest. Each successive set would be performed throughout a narrower ROM, with the $1 / 4$ ROM set resulting in the countermovement position being located only one quarter of the ROM from full elbow extension.

A potential benefit of VROM training is that the loads used during each set are modified so that each set is performed at near-maximal intensity, resulting in the mass lifted during 


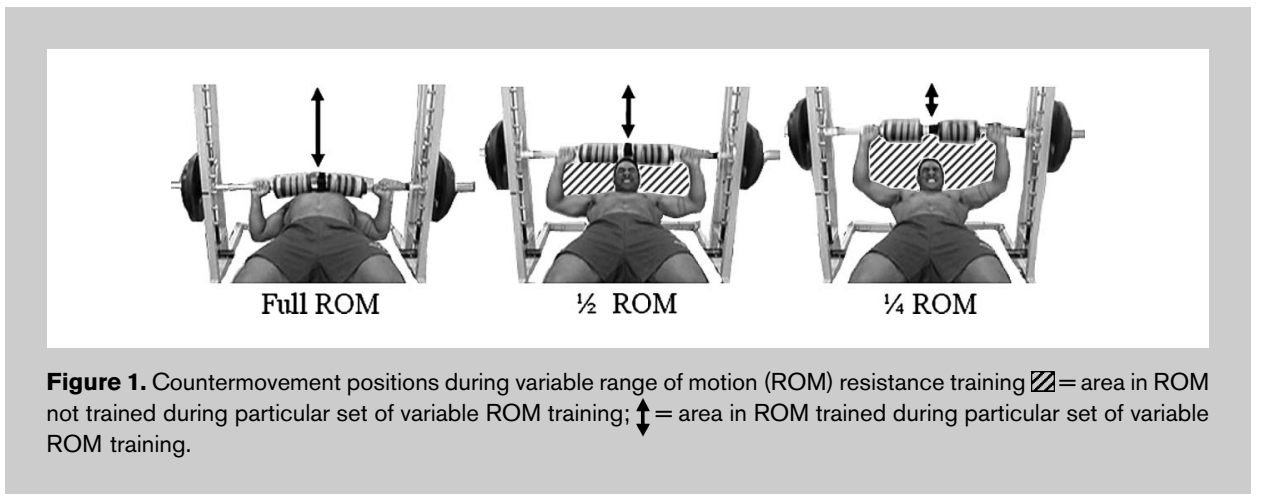

The first session consisted of $6 \mathrm{RM}$ bench press strength throughout the full and $3 / 4$ ROM from full elbow extension movements. The second session consisted of $6 \mathrm{RM}$ strength tests throughout the $1 / 2$ and $1 / 4$ ROM from full elbow extension movements. A 6RM strength test was used instead of a higher load/lower repetition maximal test because the loads during the limited ROM tests were expected to be far in excess of

the partial ROM sets far exceeding the athlete's full ROM one-repetition maximum (1RM) $(3,4,6,7)$. In addition, research suggests that performing limited ROM exercises with loads in excess of full ROM 1RM does not dramatically slow down the concentric phase of the movement (8). Consequently, if a heavier load is lifted with a similar velocity profile, force output will be increased; however, no previous research has examined this theory. Therefore, the aim of this study was to examine the peak force levels and concentric work performed during VROM training.

\section{Methods}

\section{Experimental Approach to the Problem}

In this study, we conducted 6RM strength tests for full, $3 / 4,1 / 2$, and $1 / 4$ ROM bench press and examined the load lifted, peak force, and concentric work performed during these tests.

\section{Subjects}

Six male subjects (age 20.2 \pm 1.3 years, height $179.4 \pm 4.6 \mathrm{~cm}$, weight $89.6 \pm 9.9 \mathrm{~kg}$ ) volunteered to participate in this study. The subjects were required to have a minimum of 6 months' previous resistance training experience, and all subjects participated in a 4 -week resistance training program prescribed by the primary examiner before this experiment. Based on the results of a study by Mookerjee and Ratamess (8), who also examined partial ROM bench press using five subjects and found significant results at $p<0.01$, this number of subjects would allow for high statistical power at $p<0.05$. This study received ethical approval from the Central Queensland University Human Research Ethics Committee.

\section{Procedures}

The strength testing was performed on a Smith machine (Calgym, Australia), with a digital rotary encoder (IDM Instruments, Australia) recording the position of the barbell throughout the movement. Knowledge of the mass of the bar, along with displacement and time acquired from the rotary encoder, allowed for determination of force output using custom-written Labview (National Instruments) software acquisition and analysis package sampling at $1000 \mathrm{~Hz}$.

Data collection consisted of 2 separate days of 6RM bench press strength testing, with the sessions separated by 72 hours. the load lifted during the full ROM test. Therefore, a 6RM test was chosen because it provided a test of maximal strength that did not place excessive unaccustomed strain on the subjects.

During the 4-week program leading up to this study, the subjects had performed four sessions of VROM bench press training. This was included in the subjects' training program to provide both familiarization with the testing protocol and an approximation of the loads lifted during each ROM. Although this familiarization was performed, it was still deemed necessary to split the testing into two separate sessions to prevent excessive fatigue from influencing the results of the study. Furthermore, although a crossover design was considered for testing order, the subjects' extensive familiarity with full ROM bench press resulted in this test being performed in the first session. This familiarity allowed for a reasonably accurate prediction of the subjects' 6RM full ROM strength, reducing the number of tests performed before the actual 6RM load was determined. This reduced the potential for delayed-onset muscle soreness during the second testing session.

The actual 6RM bench press strength tests were performed on the Smith machine, with metal stoppers used to restrict the vertical plane of the movement in an attempt to limit the ROM during each set. The subjects' ROM could not be exactly limited to each ROM because of the $7.5-\mathrm{cm}$ distance between stopper positions; however, this distance between settings still allowed for restrictions to the ROM that may be encountered during a typical VROM training session. Before testing, the subjects' hand positioning was determined and measured, with all tests performed using the same hand spacing position.

The tests were commenced with the subject lifting the barbell off the Smith machine supports before pressing the barbell until full elbow extension was achieved. This phase of the movement was assisted during the partial ROM tests to reduce stress on the subjects' wrist joints. During performance of the testing repetitions, the subjects were instructed to lower the barbell at a moderate pace until it reached a position slightly above the stoppers before performing a countermovement and lifting the barbell as powerfully as possible. This countermovement position resulted in no descent contact between the barbell 


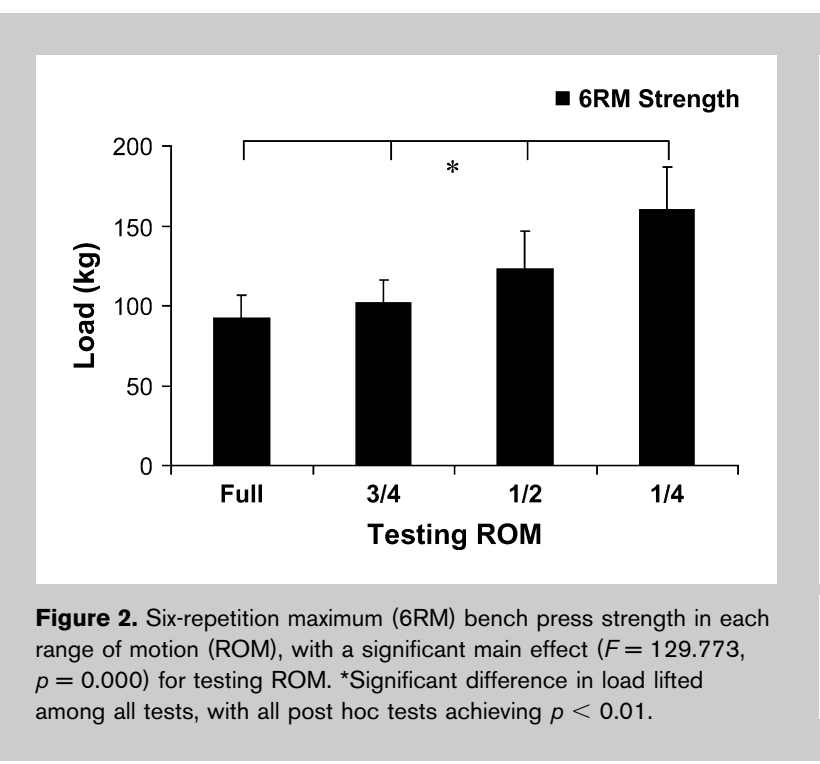

and stoppers, ensuring the integrity of elastic energy contribution during the countermovement.

An estimate of the subjects' 6RM strength in each ROM was determined based on the loads lifted during training, and these estimated loads were used as a baseline for the strength tests. A warm-up set of six repetitions using $75 \%$ of this predicted 6RM was performed before the test. If necessary, after the 6RM test was performed, the load was adjusted and repeated until the subjects' $6 \mathrm{RM}$ for that specific ROM was determined.

In addition, the concentric work performed during each repetition was examined using the formula:

$$
\begin{aligned}
& \text { Work }(N m)=\text { Mass of the external load }(N) \\
& * \text { Displacement }(m)
\end{aligned}
$$

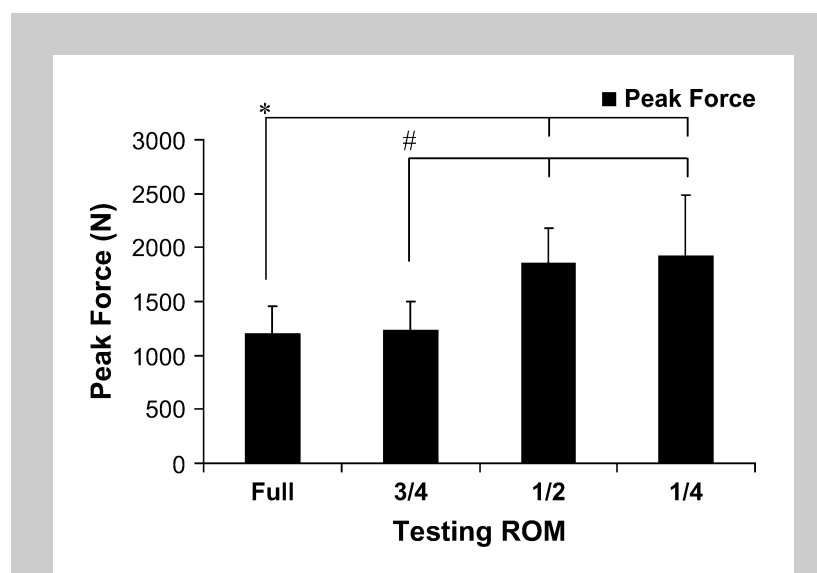

Figure 3. Peak force during six-repetition maximum (6RM) bench press in each range of motion (ROM), with a significant main effect ( $F=14.725$, $p=0.007$ ) for testing ROM. *Peak force significantly less during the full ROM test compared with the $1 / 2 \operatorname{ROM}(p=0.000)$ and $1 / 4 \operatorname{ROM}(p=$ $0.015)$ tests. \#Peak force significantly less during the $3 / 4$ ROM test compared with the $1 / 2 \operatorname{ROM}(p=0.000)$ and $1 / 4 \operatorname{ROM}(p=0.024)$ tests.

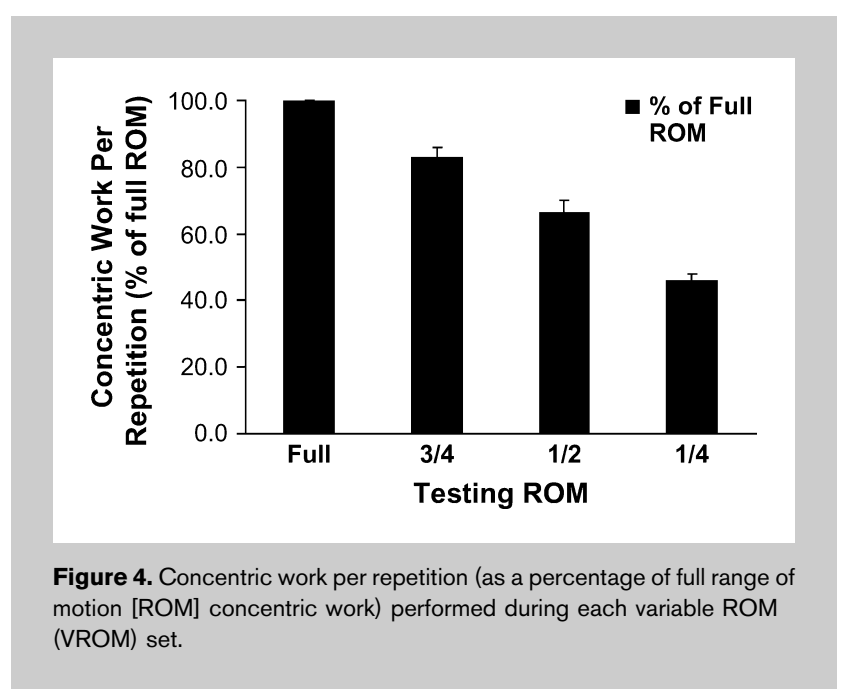

The mean value for concentric work performed per repetition was then normalized as a percentage of the concentric work performed during the full ROM bench press. This concentric work per repetition value is an important control variable for potential studies examining this method of training during a longitudinal study.

\section{Statistical Analyses}

Along with 6RM strength in each region, peak force was assessed during the concentric phase for each trial. A one-way analysis of variance was performed on the results, with an $\alpha$ level set at $p<0.05$ and Fischer's least significant difference post hoc tests performed in the event of a significant main effect or interaction.

\section{RESUlts}

The results for 6RM strength are provided in Figure 2. Analysis revealed that the load lifted during all testing sets was significantly $(p<0.05)$ different, with the load increasing as the ROM became smaller.

Peak force during each test is shown in Figure 3. Both the $1 / 2$ and $1 / 4 \mathrm{ROM}$ sets produced significantly higher peak force levels than the full and $3 / 4$ ROM tests. There were no significant differences in peak force levels between the full and $3 / 4$ or $1 / 2$ and $1 / 4$ ROM tests.

Figure 4 shows the mean concentric work per repetition performed for each VROM set. Although the load lifted increased, concentric work decreased as the ROM was restricted because of a decrease in barbell displacement.

\section{Discussion}

The results of this study reveal that both the load lifted and peak force output increase as the ROM of the bench press exercise is decreased toward terminal elbow extension. These findings are somewhat supported by the study of Mookerjee and Ratamess (8), who reported that concentric velocity did not decrease dramatically during partial ROM exercises despite an increase in the load lifted. 
These findings suggest that VROM training may help to overcome one of the major limitations of full ROM resistance training, terminal deceleration toward the end range of the movement. Although this can be reduced to some extent by incorporating ballistic exercises into the program (5), this still does not result in peak force levels occurring in the ROM where high force production is often necessary during sport. By incorporating VROM training into a resistance training program, peak force levels in excess of those that occur during full ROM training can be produced throughout a considerable segment of the midrange of the movement. Additionally, VROM training requires the performance of countermovements in different phases of the ROM during each set. This would seem to be more applicable to athletic training because most sports do not require the athlete to repeatedly perform an identical movement. Therefore, performing training throughout different phases of the ROM, with various loads, may help to optimize an athlete's performance by enhancing the true movement specificity of training. However, whether this method of training is superior to full ROM resistance training is unknown.

\section{Practical applications}

The results of this study suggest that VROM training provides superior peak force output during a resistance training session when compared with full ROM training alone.

\section{REFERENCES}

1. Bloomfield, J, Ackland, TR, and Elliott, BC. Applied Anatomy and Biomechanics in Sport. Victoria, Australia: Blackwell Scientific Publications, 1994.

2. Elliott, BC, Wilson, GJ, and Kerr, GK. A biomechanical analysis of the sticking region in the bench press. Med Sci Sports Exerc 21: 450-462, 1989.

3. Graves, JE, Pollock, ML, Jones, AE, Colvin, AB, and Leggett, SH. Specificity of limited range of motion variable resistance training. Med Sci Sports Exerc 21: 84-89, 1989.

4. Graves, JE, Pollock, ML, Leggett, S, Carpenter, D, Fix, C, and Fulton, $\mathrm{M}$. Limited range of motion lumbar extension strength training. Med Sci Sports Exerc 24: 128-133, 1992.

5. Lander, JE, Bates, BT, Sawhill, JA, and Hamill, J. A comparison between free-weight and isokinetic bench pressing. Med Sci Sports Exerc 17: 344-353, 1985.

6. Massey, CD, Vincent, J, Maneval, M, and Johnson, JT. Influence of range of motion in resistance training in women: early phase adaptations. J Strength Cond Res 19: 409-411, 2005.

7. Massey, CD, Vincent, J, Maneval, M, Moore, M, and Johnson, JT. An analysis of full range of motion training vs. partial range of motion training on the development of strength in untrained men. $J$ Strength Cond Res 18: 518-521, 2004.

8. Mookerjee, $\mathrm{S}$ and Ratamess, N. Comparison of strength differences and joint action durations between full and partial range-of-motion bench press exercise. J Strength Cond Res 13: 76-81, 1999.

9. Newton, RU, Kraemer, WJ, Häkkinen, K, Humphries, BJ, and Murphy, AJ. Kinematics, kinetics and muscle activation during explosive upper body movements. J Appl Biomech 12: 31-43, 1996.

10. Young, W, Benton, D, Duthie, G, and Pryor, J. Resistance training for short sprints and maximum speed sprints. Strength Cond J 23: $7-13,2001$ 